\title{
Momentum selectivity and anisotropy effects in the nitrogen $K$-edge resonant inelastic $x$-ray scattering from GaN
}

\author{
V. N. Strocov, ${ }^{1, *}$ T. Schmitt,,${ }^{2, \dagger}$ J.-E. Rubensson, ${ }^{2}$ P. Blaha, ${ }^{3}$ T. Paskova, ${ }^{4}$ and P. O. Nilsson ${ }^{5}$ \\ ${ }^{1}$ Paul Scherrer Institute, CH-5232 Villigen PSI, Switzerland \\ ${ }^{2}$ Department of Physics, Uppsala University, Box 530, S-75121 Uppsala, Sweden \\ ${ }^{3}$ Institut für Materialchemie, Technische Universität Wien, A-1060 Wien, Austria \\ ${ }^{4}$ Linköping University, S-581 83 Linköping, Sweden \\ ${ }^{5}$ Chalmers University of Technology, S-412 96 Göteborg, Sweden \\ (Received 6 December 2004; revised manuscript received 14 March 2005; published 31 August 2005)
}

\begin{abstract}
High-resolution soft x-ray emission and absorption spectra near the $\mathrm{N} K$-edge of wurtzite $\mathrm{GaN}$ are presented. The experimental data are interpreted in terms of full-potential electronic structure calculations. The absorption spectra, compared with calculations including core hole screening, indicate partial core hole screening in the absorption process. The resonantly excited x-ray emission spectra show pronounced dispersion of spectral structures, which is attributed to effects of momentum conservation in the resonant inelastic x-ray scattering (RIXS) process. In development of GaN based optoelectronics, momentum selectivity in RIXS can be utilized to control development of band structure in $\mathrm{GaN}$ nanostructures.
\end{abstract}

DOI: 10.1103/PhysRevB.72.085221 PACS number(s): 78.70.En, 78.55.Cr, 78.70.Dm, 71.20.Nr

\section{INTRODUCTION}

Soft-x-ray emission (SXE) and absorption (SXA) spectroscopies allow investigation of the electronic structure of the valence band $(\mathrm{VB})$ and conduction band $(\mathrm{CB})$, respectively, with elemental specificity and large probing depth up to $3000 \AA$. In particular, the electronic structure of buried quantum dots, interfaces ${ }^{1}$ and even isolated impurities ${ }^{2}$ can be accessed by these techniques (see further references in a review by Kotani and Shin). ${ }^{3}$

In general, the SXE/SXA spectroscopies characterize the electron partial density of states (PDOS) resolved in their elemental and orbital character, but averaged over the wave vectors k. For weakly correlated semiconductor systems, however, certain k-selectivity appears in resonant inelastic $\mathrm{x}$-ray scattering (RIXS) where the core electron is excited within a few $\mathrm{eV}$ above the absorption threshold. ${ }^{3}$ In this case the absorption and emission events are coupled in a fast coherent scattering process, in which the full momentum is conserved as

$$
\mathbf{q}_{\text {in }}-\mathbf{q}_{\text {out }}=\mathbf{k}_{\mathrm{e}}+\mathbf{k}_{\mathrm{h}},
$$

where $\mathbf{q}_{\text {in }}$ and $\mathbf{q}_{\text {out }}$ are wave vectors of the absorbed and emitted photons, and $\mathbf{k}_{\mathrm{e}}$ and $\mathbf{k}_{\mathrm{h}}$ are those of the conduction electron and valence hole, respectively. Dependence of the resonant SXE spectra on the excitation energy $h \nu_{\text {ex }}$ reflects then the $\mathrm{VB}$ and $\mathrm{CB}$ dispersions $E(\mathbf{k})$. The $\mathbf{k}$ selectivity of RIXS has been demonstrated for a number of semiconductors such as $\mathrm{Si}$ and $\mathrm{SiC}$, and even for the graphite semimetal. $^{3-5}$

$\mathrm{GaN}$ is a prototype nitride compound representative of a large family of binary and ternary group III nitrides, having a wide range of optoelectronic device applications covering the entire region from 200 to $1200 \mathrm{~nm}$. Presently commercial blue laser diodes are available, and lasers working in the blue-violet spectral region are realized for both pulsed and continuous wave operations. Further progress can be ex- pected in low dimensional nitride devices like quantum dot lasers or single photon emitters. The current strong interest in nanostructure systems like self-assembled $\mathrm{GaN}$ quantum dots in AlN or AlGaN matrix is motivated by their potential to achieve lasers with longer lifetimes, higher gains, lower threshold currents and improved temperature characteristics. ${ }^{6}$ Progress in fabrication of such devices is closely linked with a better knowledge about the electronic properties of the bulk nitride compounds as well as nitride based nanostructures.

Spectroscopic investigations of the GaN electronic structure are far from being exhaustive. In particular, its k-resolved studies with conventional angle-resolved photoelectron spectroscopy (ARPES) are burdened by surface effects sensitive to the sample growth and surface preparation procedures (see, e.g., Refs. 7 and 8). Moreover, small photoelectron escape depth principally limits applications of this technique to the nanostructure systems buried in a matrix of other material. The soft-x-ray spectroscopies with their large probing depth and elemental specificity are ideally suited for this purpose. In application to GaN, Stagarescu et al. ${ }^{9}$ and Lawniczak-Jablonska et al. ${ }^{10}$ used the SXE and SXA spectroscopies to study the occupied and unoccupied $\mathbf{k}$-integrated PDOS, respectively. Eisebitt et al. ${ }^{11}$ found pronounced changes in the $\mathrm{N} K$-edge SXE spectral shape occurring near the absorption threshold, which they interpreted in terms of momentum conservation. However, a systematic study of RIXS in $\mathrm{GaN}$ and its connection to the band structure is presently missing.

Here, we present a high-resolution SXE/SXA study performed on wurtzite $\mathrm{GaN}$ near the $\mathrm{N} K$-edge ( $1 s$ core level). Compared to our pilot study in Ref. 12, the present results are based on experimental data obtained for a larger set of excitation energies at higher resolution and varied scattering geometries, and receive extended theoretical analysis. We unambiguously identify effects of momentum conservation in the RIXS process. The experimental results are supported by state-of-art band structure calculations. 


\section{EXPERIMENT}

We have studied wurtzite GaN with the (0001) surface orientation. The sample was a $30 \mu \mathrm{m}$ thick GaN film grown on a sapphire substrate using hydride vapour phase epitaxy in the optimum growth window. ${ }^{13}$ High crystalline quality of the sample was confirmed by high resolution x-ray diffraction rocking curve and low temperature photoluminescence (LT-PL) measurements. The FWHM values of the $\omega$ - and $2 \theta-\omega$ radial scans for the symmetric (002) and asymmetric (102), (104), and (114) reflections approached the best values reported for thick nitride films grown on foreign substrate, and indicate relatively low mosaicity without domain formation achieved at this thickness. The LT-PL spectrum of the sample shows well resolved exciton-related peaks in the near band edge region with FWHM less than $2 \mathrm{meV}$, confirming the high crystalline and optical quality of the film.

The SXE/SXA experimental data were taken near the $\mathrm{N}$ $K$-edge $(1 s$ core level) at $\sim 400 \mathrm{eV}$. The experiments were performed at MAX-lab, Sweden, at the undulator beamline I511-3 equipped with an SX-700 type plane grating monochromator, ${ }^{14}$ and a high-resolution Rowland-mount grazing incidence SXE spectrometer. ${ }^{15}$ The incident radiation had linear $p$-polarization ( $\mathbf{E}$ vector in the incidence plane). The sample was positioned at a near-grazing (NG) incidence geometry with a grazing angle of $\sim 20^{\circ}$ relative to the incident beam.

The SXA data were measured with a monochromator resolution of $0.2 \mathrm{eV}$, which is comparable to the $\mathrm{N} 1 s$ core level intrinsic width $\sim 0.1 \mathrm{eV} .{ }^{10}$ The absorption signal was recorded in total electron yield mode and normalized to the photocurrent from a gold mesh introduced into the synchrotron radiation beam upstream the experimental chamber.

The experimental SXA spectrum is shown in Fig. 1 (right spectrum on top). In all figures the experimental data are represented in two energy scales: the one on top of the panels reflects the photon energies, whereas the one in the bottom gives the energies relative to the valence band maximum (VBM) determined by aligning the leading edges of the experimental and theoretical SXE spectra (see below).

The SXA spectrum shows significant angle dependence due to high anisotropy of GaN (Refs. 10 and 16) (see below). The spectrum in Fig. 1 measured at a NG-incidence geometry is in good agreement with the previous data ${ }^{10,16}$ measured under similar conditions, although with slight differences in the peak amplitudes due to a somewhat different incidence angle.

The SXE measurements were performed with the spectrometer installed in the incidence plane at a scattering angle of $90^{\circ}$ relative to the incident beam. With our NG-incidence geometry, the SXE spectra were therefore measured at nearnormal (NN) emission geometry with an emission angle of $\sim 20^{\circ}$ relative to the surface normal. The spectrometer was operated in 1st diffraction order with a spherical grating of 5 $\mathrm{m}$ radius and 1200 lines $/ \mathrm{mm}$ groove density, providing a resolution around $0.2 \mathrm{eV}$.

The energy scale of the spectrometer was set using the tabulated characteristic x-ray line energies of the Co $L_{\alpha}$ and $L_{\beta}$ lines from Ref. 17 recorded from a pure Co foil in 2 nd diffraction order. Based on the elastic peaks in the SXE spec-

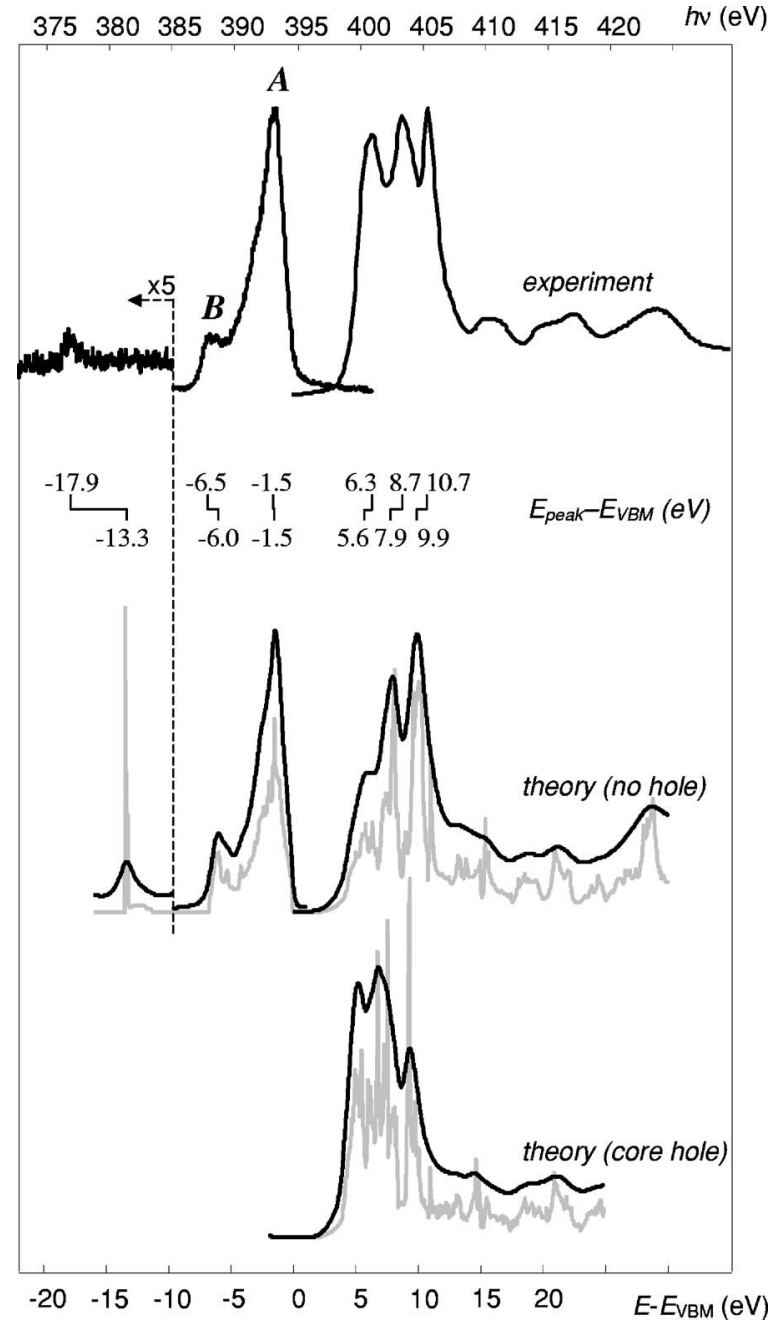

FIG. 1. (Top) Experimental N $1 s$ off-resonant SXE spectrum $\left(h \nu_{\mathrm{ex}}=428.7 \mathrm{eV}\right)$ and SXA spectrum. The energy scale on top of the panel reflects the photon energies, whereas in the one in the bottom the energies are relative to VBM. With our near-grazing incidence experimental geometry, the SXE and SXA spectra reflect mainly the $\mathrm{N} p_{\mathrm{xy}}$ PDOS in the $\mathrm{VB}$ and $\mathrm{N} p_{\mathrm{z}}$ PDOS in the $\mathrm{CB}$; (gray curves below) theoretical results excluding and including the core hole, and (superimposed black curves) empirical simulation of the lifetime and instrumental broadening effects, scaled $\times 2$; (chart between the experimental and theoretical curves) experimental energies of the SXE and three leading SXA peaks compared with the no-hole theoretical ones.

tra, this energy scale was then used to calibrate the monochromator relative to the spectrometer energy scale. This ensured the residual misfit between the spectrometer and monochromator energy scales below $0.2 \mathrm{eV}$. For further experimental details see Ref. 2.

The experimental off-resonant SXE spectrum, taken at an excitation energy $h \nu_{\mathrm{ex}}$ of $428.7 \mathrm{eV}$ well above the absorption threshold, is shown in Fig. 1 (top left spectrum). It is in good agreement with previous off-resonant data. ${ }^{9}$ Taken at $h \nu_{\text {ex }}$ well above the $\mathrm{N} K$-edge, this spectrum will further be used as a reference for the resonant series.

The experimental resonant SXE spectra are shown in Fig. 2 in comparison with the reference off-resonant one (at the 


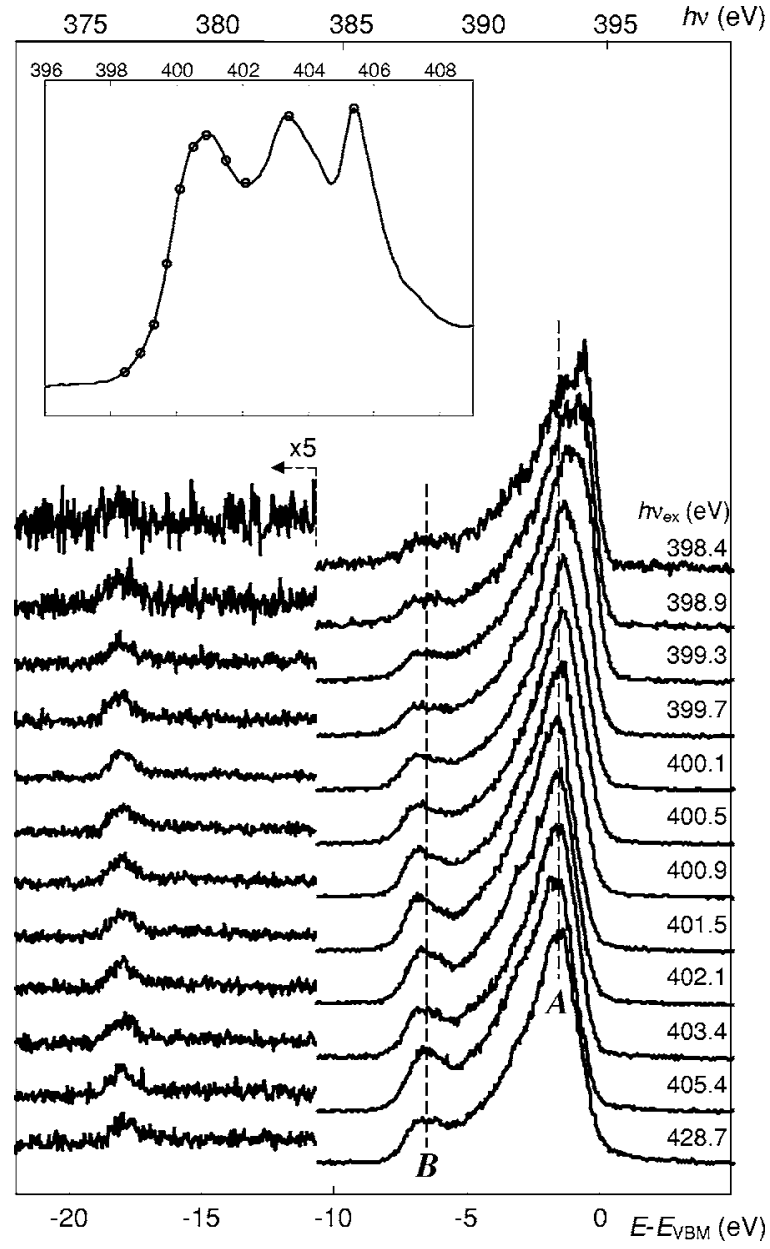

FIG. 2. Experimental N $1 s$ resonant SXE series in comparison with the off-resonant spectrum (the bottom curve), all normalized to the same peak value (relative to the foot background). The indicated excitation energies $h \nu_{\mathrm{ex}}$ are also marked at the SXA spectrum (in$s e t$ ). The off-resonant energies of the two spectral peaks are marked by dashed lines. The excitation energy dependent dispersion and line shape changes of the SXE structures are due to momentum conservation in the RIXS process.

bottom). The corresponding $h \nu_{\mathrm{ex}}$ in relation to the SXA structures (see inset of Fig. 2) are indicated on the right. The resonant spectra were measured with a monochromator resolution better than $0.2 \mathrm{eV}$, matching that of the spectrometer. The resonant SXE series shows clear dispersions and lineshape changes of both spectral peaks in the VB, with their dispersion ranges being about $0.3 \mathrm{eV}$ for the lower-energy peak, and $1.0 \mathrm{eV}$ for the higher-energy one. This identifies pronounced RIXS effects in GaN.

\section{COMPUTATIONS}

Our computations of the band structure $E(\mathbf{k})$ and PDOS were performed within the standard DFT formalism. The electron exchange-correlation was described within the generalized gradient approximation (GGA). ${ }^{18}$ The calculations employed a full-potential LAPW + Local Orbitals method $^{19,20}$ implemented in the WIEN2k package. ${ }^{21}$
This computational framework was extended to evaluate the SXA and off-resonant SXE spectra. The SXE calculations employed "the final-state rule," where the core hole is filled. ${ }^{22}$ The SXA calculations, in the first step, were performed within the "initial-state approximation," 23 where the core hole is completely neglected (for the core hole effects see below). Within these approaches the SXE/SXA spectra appear as the element and orbital projected PDOS, multiplied with the energy dependent dipole matrix elements between the core and the corresponding VB/CB states. ${ }^{24}$ In our calculations the wave function integration to evaluate the matrix elements was restricted to the atomic spheres (defined in the APW + LO method) where the core level wave functions are localized. The dipole matrix elements usually appear as quite smooth functions of energy and do not much alter the corresponding PDOS. (This is different when one has a $p$-type core hole, e.g., an $L_{2,3}$ spectrum, where the contributions of the $p \rightarrow s$ and $p \rightarrow d$ channels crucially depend on the ratio of the corresponding matrix elements.)

Due to the anisotropy of GaN, the PDOS and SXE/SXA computations were performed for the $\mathrm{N} p_{\mathrm{z}}$ and $\mathrm{N} p_{\mathrm{xy}}$ orbitals separately. The SXE/SXA spectra relevant for our experimental geometry were obtained by summation of the $\mathrm{N} p_{\mathrm{z}}$ and $\mathrm{N} p_{\mathrm{xy}}$ spectra weighted by relative squared amplitudes of the $\mathbf{E} \| \mathbf{c}$ and $\mathbf{E} \perp \mathbf{c}$ components of the $\mathbf{E}$ vector (see below).

The calculated SXE/SXA spectra are shown in Fig. 1 in comparison with the experiment. The smooth curves include an empirical simulation of the energy dependent lifetimes of the valence band hole and conduction band electron. This was achieved by convolution of the calculated spectra with a Lorentzian, whose full width was heuristically taken to depend on energy in proportion to the energy distance from the Fermi level (taken in the middle of the band gap) as $\Gamma$ $=\alpha\left|E-E_{\mathrm{F}}\right|$. Additional broadening due to the $\mathrm{N} 1 s$ core level width of $\sim 0.1 \mathrm{eV}$ was insignificant. The experimental resolution was also included into this simulation by additional Gaussian convolution. By comparison of the simulated spectral broadening with the experiment, we estimated the proportionality coefficient to be $\alpha \sim 0.1$. This gives a rough estimate for the energy dependence of lifetimes through the VB and CB. The simulated SXE spectrum was used to set the VBM energy position in the experimental SXE spectra by aligning the spectral leading edges.

The theoretical SXE/SXA spectra in Fig. 1 demonstrate, on the whole, convincing agreement with the experiment. Even such a subtle detail as a structure on the low-energy slope of the dominant SXE peak is well reproduced. The agreement extends up to $30 \mathrm{eV}$ above the absorption threshold. Some energy shifts between the theoretical and experiment spectral structures are due to excited-state self-energy corrections, and relative amplitude disagreements in SXA can be attributed to core hole effects (see below).

Our computational results are a major improvement compared to the previous LMTO calculations from Ref. 16, which were restricted by the use of the muffin-tin (MT) potential. This illustrates the importance of full-potential effects in covalent materials such as GaN. Further computational results are presented in the section below. 


\section{RESULTS AND DISCUSSION}

\section{A. SXA and off-resonant SXE}

\section{Overview of the experimental spectra}

The experimental $\mathrm{N} 1 s$ SXA spectrum in Fig. 1 reflects the $p$-projected PDOS in the $\mathrm{N}$ core region through the $\mathrm{CB}$, This is because the dipole selection rules require that the orbital angular momentum quantum number $l$ changes by \pm 1 in the process of photon absorption or emission. In fact, due to predominance of the antibonding $2 p$ states in the CB of $\mathrm{GaN}$ this PDOS is almost equivalent to the total density of states (DOS). With the fundamental band gap of $\mathrm{GaN}$ being $3.4 \mathrm{eV}$, the SXA spectral onset is placed $\sim 1 \mathrm{eV}$ above the conduction band minimum (CBM), where the PDOS vanishes (see below).

The experimental SXE spectrum in Fig. 1 reflects, by the same selection rules, the $p$-projected PDOS in the $\mathrm{N}$ core region for the occupied states. In the photon energy region from 387 to $395 \mathrm{eV}$, the spectrum reflects the VB composed mostly of the bonding $\mathrm{N} 2 p$ states. It shows here two peaks, which are further referred to as $A$ for the main one and $B$ for the minor one at lower energy.

A small peak in the SXE spectrum near $377.5 \mathrm{eV}$ was first noticed in Ref. 9 (although we find it $1.6 \mathrm{eV}$ higher in energy, which may be traced back to different x-ray lines used in calibration). It was interpreted as due to hybridization of the Ga $3 d$ semicore states with $\mathrm{N} 2 p$ states. The Ga $3 d$ origin of this SXE peak is consistent with the binding energy position of the Ga $3 d$ peak in the ARPES spectra of GaN by Dhesi et al. ${ }^{6}$ It should be noted, however, that the hybridization with $\mathrm{N} 2 p$ states is not crucial for the Ga $3 d$ states to appear in the $\mathrm{N} 1 s$ SXE spectrum: If they merely protrude into the $\mathrm{N}$ core region without hybridization, their angular momentum expansion from the $\mathrm{N}$ site will always contain some $p$ component. This picture is confirmed by our band structure computations, which indicate negligible hybridization of the Ga $3 d$ and $\mathrm{N} 2 p$ states. Interestingly, the onset of the Ga $3 d$ derived structure with $h \nu_{\text {ex }}$ (Fig. 2) seems to be slightly delayed compared to the main $\mathrm{N} 2 p$ region.

On the high-energy side of the experimental Ga $3 d$ peak we can distinguish a weak but statistically significant plateau, extending by $\sim 5 \mathrm{eV}$ to higher energy. It is not reproduced by our calculations restricted by the dipole approximation. In an ARPES study of GaN, Dhesi et al. ${ }^{6}$ also observed a weak structure on the high-energy side of the Ga $3 d$ peak, and interpreted it as due to the $\mathrm{N} 2 s$ states. Although in SXE these states are forbidden by the dipole selection rules, some intensity can in principle appear through non-dipole transitions.

\section{Anisotropy effects}

In $\mathrm{GaN}$ the $\mathrm{VB}$ and $\mathrm{CB}$ are dominated by the $\mathrm{N} 2 p_{\mathrm{z}}$ and $2 p_{\mathrm{xy}}$ orbitals, oriented, parallel and perpendicular to the (0001) surface normal $\mathbf{c}$, respectively. The selection rules in SXA are such that the excitation cross section into a particular $p$ orbital is maximized if the incident $\mathbf{E}$ vector is oriented parallel to the orbital axis, and vanishes if perpendicular. ${ }^{25}$ Therefore, the $\mathbf{E} \| \mathbf{c}$ component of the $\mathbf{E}$ vector excites the $p_{\mathrm{z}}$ orbitals, and the $\mathbf{E} \perp \mathbf{c}$ component the $p_{\mathrm{xy}}$ ones. For our ex-

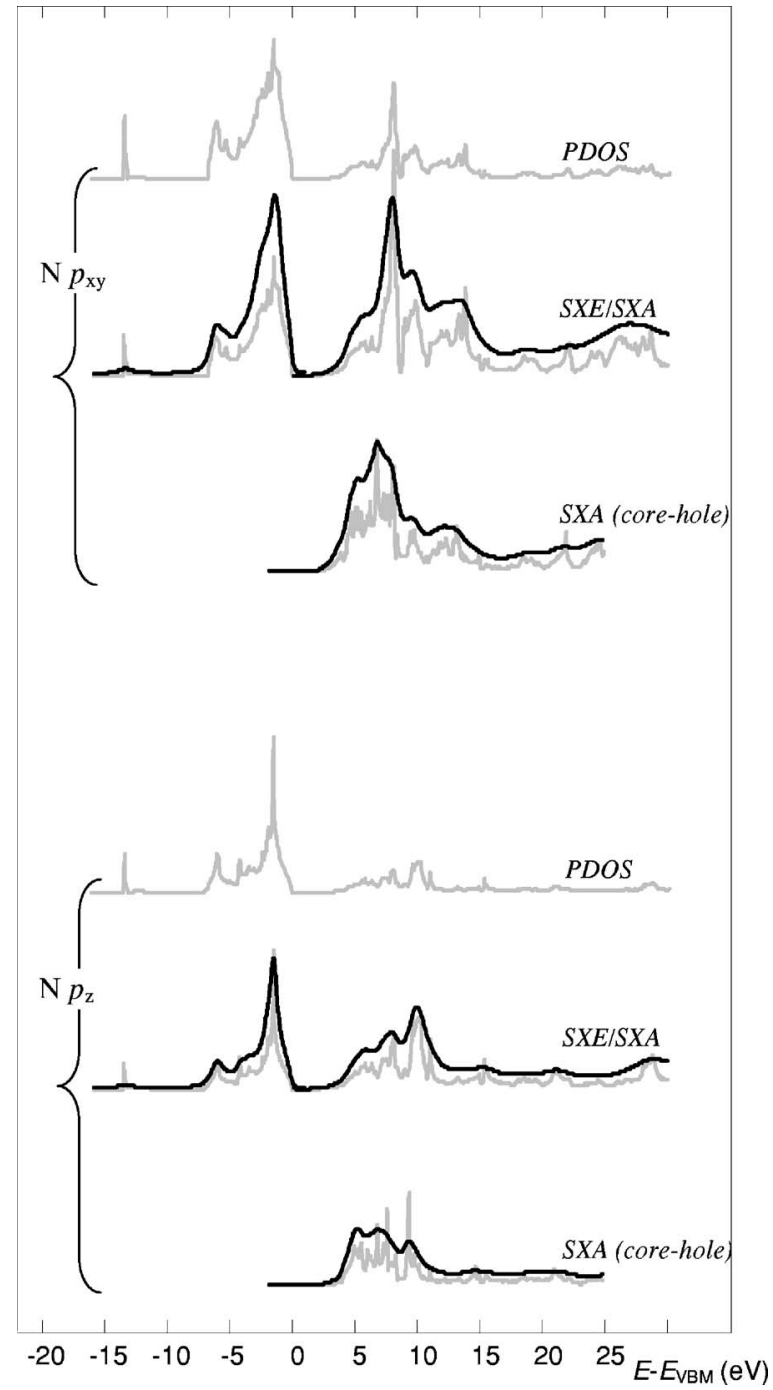

FIG. 3. (Top curves) Theoretical $\mathrm{N} p_{\mathrm{xy}}$ PDOS with the corresponding normal emission SXE spectrum, and normal incidence SXA spectrum (excluding and including core hole); (bottom) $\mathrm{N} p_{\mathrm{z}}$ PDOS with the corresponding grazing emission SXE and grazing incidence SXA spectra. Difference between the $\mathrm{N} p_{\mathrm{xy}}$ and $\mathrm{N} p_{\mathrm{z}}$ PDOS results in angle dependence of the SXE/SXA spectra. The lifetime and instrumental broadening is simulated as in Fig. 1.

perimental SXA spectrum taken with a grazing incidence angle of $20^{\circ}$, the ratio between $(\mathbf{E} \| \mathbf{c})^{2}$ and $(\mathbf{E} \perp \mathbf{c})^{2}$, representing the relative weight of the $\mathrm{N} p_{\mathrm{z}}$ and $\mathrm{N} p_{\mathrm{xy}}$ contributions to the SXA spectrum, is about 7.5:1. Therefore, our SXA spectrum taken at a NG-incidence geometry reflects mainly the unoccupied $\mathrm{N} p_{\mathrm{z}}$ orbitals. The same selection rules apply to SXE. For our experimental SXE spectrum the ratio between the $\mathrm{N} p_{\mathrm{x}}$ and $\mathrm{N} p_{\mathrm{xy}}$ contributions of about 1:7.5, is reverse to the SXA case. Therefore, our SXE spectrum taken at a $\mathrm{NN}$-emission geometry reflects mainly the occupied $\mathrm{N} p_{\text {xy }}$ orbitals.

In highly anisotropic wurtzite GaN, the PDOS associated with the $\mathrm{N} p_{\mathrm{z}}$ and $\mathrm{N} p_{\mathrm{xy}}$ orbitals is significantly different. This is illustrated by computations in Fig. 3. The SXE/SXA spectra are seen to be essentially the PDOS multiplied by the dipole matrix element with weak energy dependence. The 
difference of the $\mathrm{N} p_{\mathrm{z}}$ and $\mathrm{N} p_{\mathrm{xy}}$ PDOS, replicated by differences of the corresponding grazing and normal incidence or emission spectra, gives rise to the SXE/SXA angle dependence. Dramatic differences of the $\mathrm{N} p_{\mathrm{z}}$ and $\mathrm{N} p_{\mathrm{xy}}$ PDOS in the $\mathrm{CB}$ result in a particularly strong angle dependence of the SXA spectra. ${ }^{10,16}$ The theoretical SXE/SXA spectra in Fig. 1, corresponding to our experimental geometry, were obtained as a superposition of the $\mathrm{N} p_{\mathrm{z}}$ and $\mathrm{N} p_{\mathrm{xy}}$ spectra weighted according to the above $(\mathbf{E} \| \mathbf{c})^{2}$ to $(\mathbf{E} \perp \mathbf{c})^{2}$ ratios.

\section{Core hole effects in SXA}

The core hole generated in the SXA process in fact dynamically interacts with the valence electrons and modifies the crystal potential around it where the conduction electron is placed (see, e.g., Ref. 26). Of the static approximations to this process, ${ }^{22,23}$ one limit is the "initial-state approximation," where the core hole is completely ignored. It was implied by the above SXA calculations. The other limit is the "final-state rule," which considers a crystal potential resulting from static screening of the core hole by valence electrons.

Our SXA computations were further extended to include the core hole within the "final-state rule." A self-consistentfield (SCF) potential was generated in a supercell with the core hole on a probe atom. The computations employed a $2 \times 2 \times 1$ supercell $(16$ atoms/cell) with one $1 s$ core hole on one of the $\mathrm{N}$ atoms. The missing core electron was added to the valence electrons. During the SCF-cycle all states (of the atom where the core hole resides and of all neighbor atoms) were allowed to respond to this core hole (a larger "effective nuclear charge") and participate in the screening.

The $\mathrm{N} p_{\mathrm{z}}$ and $\mathrm{N} p_{\mathrm{xy}}$ SXA spectra calculated within the "final-state rule" are shown in Fig. 3, and their superposition corresponding to our experimental geometry in Fig. 1. Compared to the "initial-state approximation," the spectral structures slightly shift to lower energies. This reflects excitonic coupling between the screened core hole and conduction electron. The shifts show non-monotonous energy dependence, which identifies dependence of the core hole effects on the character of the CB states. Relative amplitudes of the spectral structures are changed dramatically. In particular, the amplitude of the first SXA peak in the "initial-state approximation" appears underestimated compared to the experiment, whereas the screened core hole results in an overestimated amplitude. The opposite holds for the third SXA peak. The experimental amplitudes appear somewhere between the "initial-state" and "final-state" limits, which suggests partial core hole screening, ${ }^{22}$ implying significant mobility of the valence charge in GaN. Previously it was shown that "optimization" of the theoretical spectra by choosing a partial core hole (e.g., half a $1 s$ electron) may lead to better agreement with experiment. ${ }^{22}$ However, here we refrained from such an approach because of its empirical character.

\section{Excited-state self-energy corrections}

In SXE, as far as the "final-state rule" originally derived for simple metals ${ }^{22}$ holds, the spectral structures reflect the quasi-particle energy levels of the valence hole in the $N-1$ electron system left behind after the radiative deexcitation. Their shifts from the DFT theoretical energy levels are the self-energy corrections, $\Delta \Sigma$, appearing due to difference in the exchange-correlation potential between the excited and ground state. Difference of our experimental and theoretical peak energies (see the chart in Fig. 1) yields $\Delta \Sigma$ values of $-0.1,-0.5$, and $-4.6 \mathrm{eV}$ for the $A, B$, and $\mathrm{Ga} 3 d$ derived peaks, respectively. As expected, $\Delta \Sigma$ increases when going away from the Fermi level, and is particularly large for the Ga $3 d$ derived peak due to the localized semicore character of this state.

In SXA, the $\Delta \Sigma$ corrections reflecting quasi-particle energy levels of the conduction electron in $N+1$ electron system are distorted by excitonic interaction with the partially screened core hole. The interaction energy can be estimated, roughly, as half the energy difference between the SXA peaks calculated with and without the core hole. With such a correction, the shifts between the experimental and theoretical peaks in Fig. 1 yield $\Delta \Sigma$ values of $+0.9,+1.4$, and $+1.1 \mathrm{eV}$ for the three dominant SXA peaks, in the order of increase in energy. Their sign is consistent with the band gap problem of the DFT, which neglects the exchange-correlation discontinuity for excitations across the band gap. However, their magnitude shows nonmonotonous energy dependence and, moreover, appears reduced compared to the $+1.5 \mathrm{eV}$ shift in the CBM (difference between the experimental optical band gap of $3.4 \mathrm{eV}$ and the DFT one of $1.9 \mathrm{eV}$ ). Similarly to the core hole effects, such an anomalous behavior of $\Delta \Sigma$ may indicate dependence of the self-energy effects on the character of the $\mathrm{CB}$ states. ${ }^{27}$

\section{B. RIXS}

\section{Energy dependence of the coherent fraction}

Changes in the experimental resonant SXE series within the VB region are emphasized in difference spectra shown in Fig. 4 (top). These spectra are obtained from the curves in Fig. 2 by subtracting the off-resonant reference spectrum. The latter was scaled, for each spectrum, to set the difference spectrum positive and having its minimal value equal to zero. To reduce the influence of noise on this procedure, the scaling coefficient was determined from the spectra, which had been denoised with Gaussian smoothing.

The difference spectra in Fig. 4 reflect the coherent fraction in the resonant SXE spectra: If the off-resonant spectrum - by virtue of large phase space available at high excitation energies for electron-phonon $(e-p h)$ and electronelectron $(e-e)$ interactions with the conduction electron in the intermediate state, which results in effective averaging over $\mathbf{k}$ space-represents the incoherent fraction, the positive intensity remaining after its subtraction is the coherent fraction. ${ }^{4}$ The difference spectra show two dispersing peaks.

The relative weight of the coherent fraction in the total SXE spectra within the VB as a function of $h \nu_{\mathrm{ex}}$ is shown in Fig. 5. They were evaluated as integrals of the difference spectra from Fig. 4 over the VB energy interval from 387.4 to $395.4 \mathrm{eV}$, divided by corresponding integrals of the total spectra from Fig. 2. The $h \nu_{\text {ex }}$ dependence of the latter is in fact equivalent to the SXA spectrum, also shown in Fig. 5, 
$385387389391393395397399401403405 \quad h v(\mathrm{eV})$
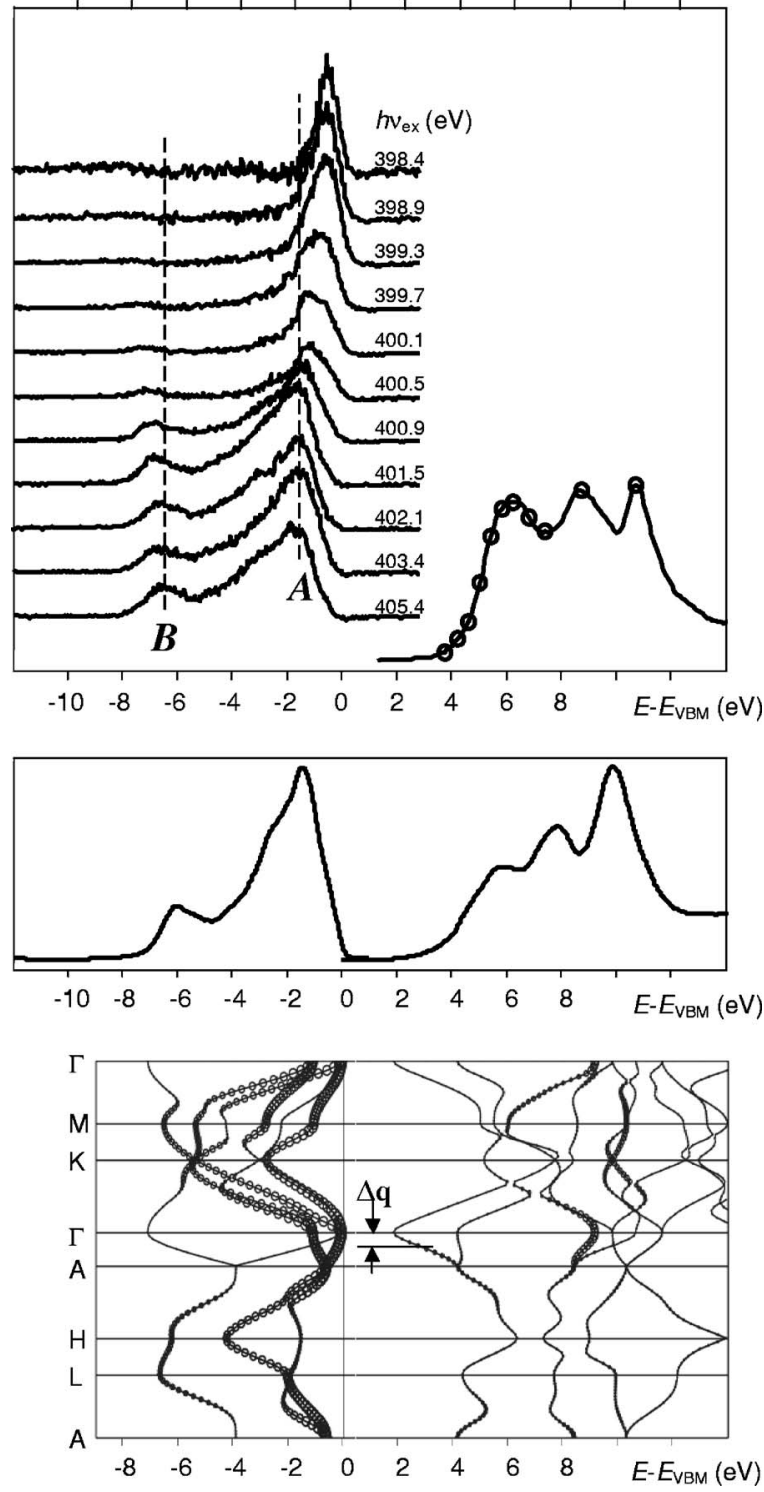

FIG. 4. (Top) Differential resonant SXE spectra, representing the coherent spectral fraction, normalized to the same intensity maximum. The indicated excitation energies $h \nu_{\mathrm{ex}}$ are also marked at the SXA spectrum. The energies of the two off-resonant SXE spectral peaks are marked by dashed lines; (middle) calculated $\mathrm{N} p_{\mathrm{xy}}$ derived SXE and $\mathrm{N} p_{\mathrm{z}}$ derived SXA spectra; (bottom) calculated $E(\mathbf{k})$. The weights of the $\mathrm{N} 2 p_{\mathrm{xy}} \mathrm{VB}$ states and $\mathrm{N} 2 p_{\mathrm{z}} \mathrm{CB}$ states are indicated by the radii of the circles. Dispersion of the resonant SXE spectral structures is related primarily to that of the $\mathrm{N} 2 p_{\mathrm{xy}}$ valence states and $\mathrm{N} 2 p_{\mathrm{z}}$ conduction states.

because the $\mathrm{N} 1 s$ core hole can be radiatively filled only from the $\mathrm{N} 2 p$ VB states (the contribution from the Ga $3 d$ derived states is negligible).

The coherent fraction in Fig. 5 vanishes at higher energies because the strength of the $e-p h$ and $e-e$ interaction, increases with energy due to increase of the phase space available for them. However, below $h \nu_{\text {ex }}$ of $405 \mathrm{eV}$ our experimental data demonstrate, surprisingly, that the coherent fraction weight behaves non-monotonously and even increases with energy. This fact is beyond the above simple

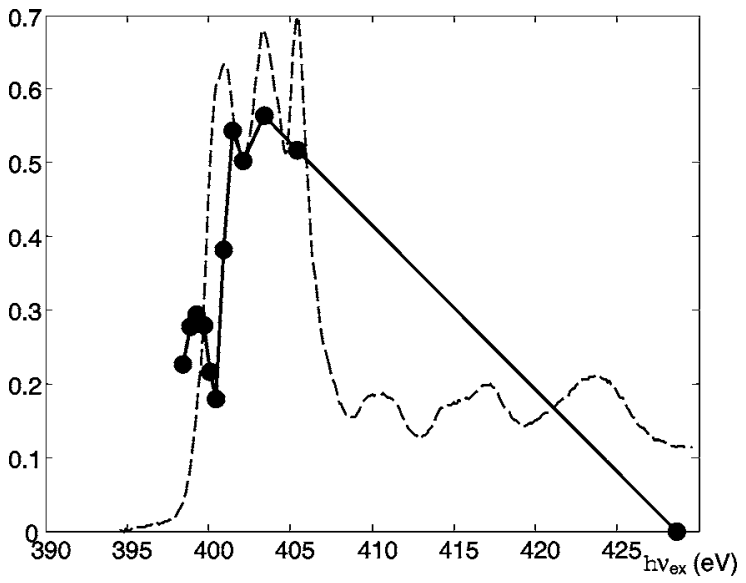

FIG. 5. Relative weight of the coherent SXE fraction as a function of excitation energy (the thin line connecting the dots is a guide for the eye) compared with the SXA spectrum (dashed line, arbitrarily scaled) reflecting the total SXE intensity. Anomalous behavior of the coherent fraction at low $h \nu_{\mathrm{ex}}$ reflects changes in character of the conduction states.

phase space arguments. Evidently, the low-energy conduction band states in $\mathrm{GaN}$ change their character in this energy region in such a way that their susceptibility to the incoherent $e-p h$ and $e-e$ scattering varies. Tentatively, we attribute this effect to the anisotropy of the material. As the coherent fraction is larger in the region where the CB layer-projected DOS is dominated by $\mathrm{N} p_{\mathrm{xy}}$ states, it suggests that the incoherent scattering is in some way less pronounced within the atomic layers than between the layers. Interestingly, the coherent fraction remains large up to rather high excitation energies.

\section{k-selectivity effects}

Our experimental SXE and SXA spectra, taken in a NNemission/NG-incidence geometry, are related primarily to the VB states having the $\mathrm{N} p_{\mathrm{xy}}$ character and CB states having $\mathrm{N}$ $p_{\mathrm{z}}$ character. Figure 4 (bottom) shows the calculated $E(\mathbf{k})$ where the $\mathrm{N} 2 p_{\mathrm{xy}}$ weights of the VB states and $\mathrm{N} 2 p_{\mathrm{z}}$ of the $\mathrm{CB}$ states are indicated. As a link between the experimental spectra and calculated $E(\mathbf{k})$, Fig. 4 (middle) reproduces the calculated off-resonant $\mathrm{N} p_{\mathrm{xy}} \mathrm{SXE}$ and $\mathrm{N} p_{\mathrm{z}}$ SXA spectra from Fig. 3, which are essentially equivalent to the corresponding PDOS. $E(\mathbf{k})$ of $\mathrm{GaN}$ appears rather involved, featuring a multitude of critical points (including those from non-symmetry Brillouin zone directions) with energy separation smaller than the lifetime broadening. The peaks in the off-resonant SXE (and SXA) spectra can therefore not be related to certain critical points. Such a relation appears only for the resonant SXE spectra due to k-selectivity effects in RIXS.

The following k-selectivity effects are identified in the resonant SXE series in comparison with the calculated $E(\mathbf{k})$.

(1) When $h \nu_{\mathrm{ex}}$ is tuned to the absorption onset at 398.4 $\mathrm{eV}$, the SXE spectrum (Fig. 2) shows a peak splitting off on the high energy side of the main peak. This effect is related to the VBM at the $\Gamma$ point: At the absorption onset, as seen 
by comparison of the calculated SXA spectrum to $E(\mathbf{k})$, the excited conduction electrons appear already in some $1 \mathrm{eV}$ above the CBM (the onset is delayed relative to the CBM because the lowest $\mathrm{CB}$ states, depleted in the $\mathrm{N} p$ character and having rather steep dispersions, deliver too small PDOS). The lowest conduction band puts the corresponding $\mathbf{k}$-vectors in some $0.3 \AA^{-1}$ from the $\Gamma$ point near the $\Gamma \AA$ direction, where this band acquires significant $\mathrm{N} p_{\mathrm{z}}$ character. The photon momentum transfer $\Delta \mathbf{q}=\mathbf{q}_{\text {out }}-\mathbf{q}_{\text {in }}$ in our experiment has almost the same value $0.28 \AA^{-1}$, and is also directed close to $\Gamma$ A. Therefore, as indicated in the $E(\mathbf{k})$ panel, the $\mathbf{k}$-conserving RIXS process couples the excited $\mathrm{N} p_{\mathrm{Z}}$ conduction electrons to the $\mathrm{N} p_{\mathrm{xy}}$ valence holes in the VBM at the $\Gamma$ point, which results in a coherent emission peak from the VBM appearing on the high energy side of the main peak.

The VB bottom is placed in the same $\Gamma$ point as the VBM. Therefore, at the absorption onset it also gives coherent emission, most clearly seen in the difference spectra (Fig. 4) as an intensity enhancement near the binding energy of $-8.2 \mathrm{eV}$. Taking self-energy effects into account, this figure is consistent with the theoretical VB bottom at $-7.1 \mathrm{eV}$.

It should be noted that due to relatively high photon energies at the $\mathrm{N} K$-edge the proper interpretation of RIXS requires that non-negligible photon momentum transfer is taken into account.

(2) As $h \nu_{\text {ex }}$ increases, the main peak in the difference spectra disperses to lower energies. In the total SXE spectra, this makes the main peak disperse towards the off-resonant position $A$. This effect is related to the band dispersion around the $\Gamma$ point: With increase of $h \nu_{\mathrm{ex}}$, the conduction band electron $\mathbf{k}$-vector moves away from $\Gamma$, following the $2 p_{\mathrm{z}}$ conduction band along the $\Gamma \mathrm{AH}$ line. Reflecting the $2 p_{\mathrm{xy}}$ heavy-hole valence bands along $\Gamma A H$ (the light-hole band does not contribute due to its $p_{\mathrm{z}}$ character in this region of the $\mathbf{k}$ space), the main coherent peak disperses then to lower energies. Its dispersion range towards $h \nu_{\mathrm{ex}} \sim 401 \mathrm{eV}$, where it passes the off-resonant position, is $\sim 0.9 \mathrm{eV}$. This figure well matches the theoretical CB and VB dispersions. We do not detect any dispersion renormalization due to core hole effects. It should be noted that the GaN anisotropy is crucial in this picture, allowing us to disentangle various bands in $E(\mathbf{k})$ based on the orbital orientation selectivity.

In principle, pushing $h \nu_{\mathrm{ex}}$ below the $398.4 \mathrm{eV}$ shifts the conduction electron towards the CBM, and the valence hole, coupled by the $\Delta \mathbf{q}$ momentum transfer, away from the VBM. This should result in shifting of the main coherent peak again to lower energies. However, the experiment in this $h \nu_{\mathrm{ex}}$ region is difficult due to vanishing absorption.

(3) With $h \nu_{\text {ex }}$ varied near $401 \mathrm{eV}$, the difference spectra display a minor coherent peak dispersing below the incoherent one $B$ at $-6.5 \mathrm{eV}$. Based on the $\mathrm{N} p_{z}$ dispersion in the $\mathrm{CB}$, this can be associated with the $\mathrm{N} p_{\mathrm{xy}}$ dispersion in the $M$ valley near the VB bottom.

(4) Upon further increase of $h \nu_{\mathrm{ex}}$ above $401 \mathrm{eV}$, the main coherent peak moves below the incoherent one $A$. Its energy position can be associated with an integral effect of a few critical points in $\sim 2 \mathrm{eV}$ below the VBM such as that in the $\mathrm{M}$ point.

RIXS phenomena have also been observed for isolated $\mathrm{N}$ impurities in GaAsN. ${ }^{2}$ These phenomena were interpreted in

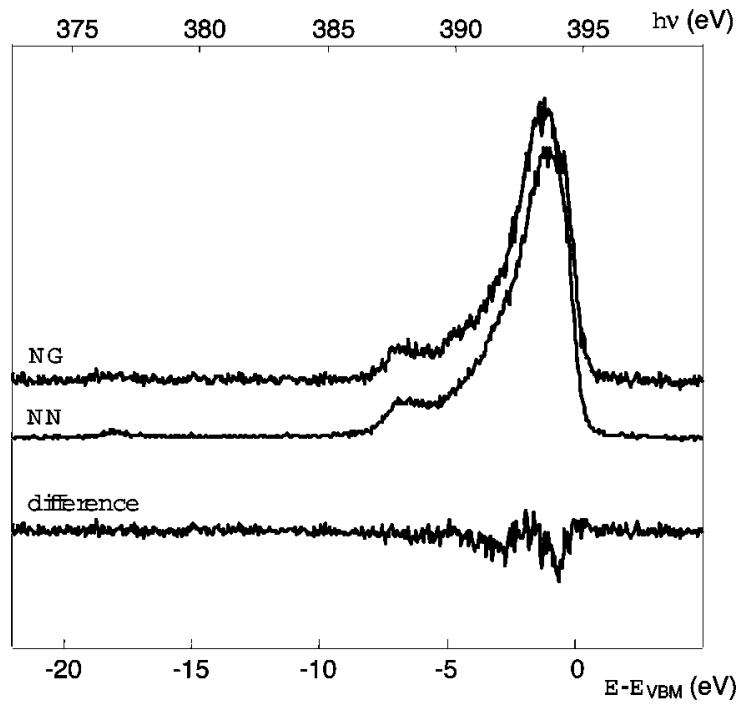

FIG. 6. Resonant SXE spectrum measured with $h \nu_{\mathrm{ex}}$ $=399.3 \mathrm{eV}$ at a NG-emission geometry (top curve) compared to its $\mathrm{NN}$-emission counterpart from Fig. 2 normalized to the same peak value (below), and their difference (bottom). The difference between the spectra is due to the difference between the $\mathrm{N} p_{\mathrm{xy}}$ and $\mathrm{N}$ $p_{\mathrm{z}}$ states.

terms of remnant k-conservation using spectral decomposition $^{28}$ of localized $\mathrm{N}$ wave functions over the Bloch waves of bulk GaAs.

\section{Emission angle dependence}

The above discussion involving the anisotropy effects suggests that the resonant SXE spectra should show certain dependence on the emission angle. To check this, we rotated the sample towards a $\mathrm{NN}$-incidence or NG-emission geometry with a grazing emission angle of $20^{\circ}$, opposite to the experimental geometry above. In this case the absorption is mainly due to the unoccupied $\mathrm{N} p_{\mathrm{xy}}$ orbitals and emission due to the occupied $\mathrm{N} p_{\mathrm{z}}$ states. However, the SXE signal in this geometry gets strongly suppressed due to the selfabsorption as well as the larger distance between the spot and spectrometer slits. We have therefore measured only one representative resonant SXE spectrum with $h \nu_{\mathrm{ex}}=399.3 \mathrm{eV}$ not far from the absorption threshold.

The experimental NG-emission spectrum is shown in Fig. 6 compared with the NN-emission one from Fig. 2 measured at the same $h \nu_{\mathrm{ex}}$. Their difference is plotted below. The spectra show statistically significant differences, reflecting the difference between the $\mathrm{N} p_{\mathrm{xy}}$ and $\mathrm{N} p_{\mathrm{z}}$ states. In particular, in the NG-emission spectrum the spectral maximum is shifted by $\sim 0.15 \mathrm{eV}$ to deeper energies. This reflects more steep dispersion of the valence $\mathrm{N} p_{\mathrm{z}}$ states away from the $\Gamma$-point compared to the $\mathrm{N} p_{\mathrm{xy}}$ states.

\section{Outlook: Nitride nanostructures}

A number of recent investigations in the field of nitride compounds optoelectronics are focused on nanostructures such as $\mathrm{Ga}(\mathrm{Al}) \mathrm{N}$ quantum dots (for a recent review, see Ref. 6). It is expected that quantum confinement effects and 
smaller defect densities achieved in nanostructures would lead to higher optical efficiency compared to bulk materials. They are also considered as potential UV-light emitters. Of particular interest are $\mathrm{Ga}(\mathrm{Al}) \mathrm{N}$ nanocolumns, which can be grown with a diameter of $30-150 \mathrm{~nm}$ and heights up to $1.6 \mu \mathrm{m}$ on different substrates such as $\mathrm{Si}(111)$ or $\mathrm{Al}_{2} \mathrm{O}_{3}$ (with or without buffer layer, typically AlN having a larger band gap). ${ }^{29}$ Soft-X-ray spectroscopies with their large probing depth (up to $300 \mathrm{~nm}$ ) and elemental specificity are ideally suited to investigate the electronic structure of such nanostructures. Grazing incidence experimental geometry can be used to stay away from the $\mathrm{N}$ signal of the buffer layer. The k-selectivity in RIXS from GaN will allow for characterization of the band structure in such nanostructures with resolution in $\mathbf{k}$-space (with description in terms of $\mathbf{k}$ applicable to the confined electron states in the sense of spectral decomposition of wave functions ${ }^{28}$ over the Bloch waves of bulk $\mathrm{GaN}$ ) and its development with diameter and heights of nanocolumns. Other interesting objects, although not yet practically realized, are GaN nanotubes. ${ }^{30}$ They are predicted to behave as direct band gap semiconductors when zigzag shaped, and indirect when armchair shaped. RIXS can be used to discriminate direct vs. indirect character of such nanostructures.

\section{SUMMARY AND CONCLUSIONS}

High-resolution SXE/SXA experimental data on wurtzite $\mathrm{GaN}$ near the $\mathrm{N} K$ edge are presented. The measurements are supported by full-potential calculations extended to the core hole screening.

The obtained results identify, in particular the following: (1) partial core hole screening in the SXA process; (2) effects of the GaN anisotropy in the SXE/SXA processes. Our experimental geometry invoked primarily the $p_{\mathrm{xy}}$ states in the $\mathrm{VB}$ and $p_{\mathrm{z}}$ states in the $\mathrm{CB}$; (3) pronounced dispersions of the resonant SXE structures, identifying the effects of momentum conservation and k-selectivity in the RIXS process; (4) nonmonotonous behavior of the coherent SXE fraction, reflecting different effects of the involved $\mathrm{CB}$ states in the $e-e$ and $e-p h$ scattering.

The $\mathbf{k}$ selectivity and anisotropy effects in RIXS can be utilized as an advanced tool to control development of band structure in $\mathrm{GaN}$ based nanostructures, allowing optimization of the shape, size and density of the nanostructure organization for particular applications.
*Corresponding author. Email address: vladimir.strocov@psi.ch

${ }^{\dagger}$ Corresponding author. Present address: Swiss Light Source, Paul Scherrer Institute, CH-5232 Villigen PSI, Switzerland. Email address: thorsten.schmitt@psi.ch

${ }^{1}$ P. O. Nilsson, J. Kanski, J. V. Thordson, T. G. Andersson, J. Nordgren, J. Guo, and M. Magnuson, Phys. Rev. B 52, R8643 (1995).

${ }^{2}$ V. N. Strocov, P. O. Nilsson, T. Schmitt, A. Augustsson, L. Gridneva, D. Debowska-Nilsson, R. Claessen, A. Yu. Egorov, V. M. Ustinov, and Zh. I. Alferov, Phys. Rev. B 69, 035206 (2004).

${ }^{3}$ A. Kotani and S. Shin, Rev. Mod. Phys. 73, 203 (2001).

${ }^{4}$ J. Lüning, J.-E. Rubensson, C. Ellmers, S. Eisebitt, and W. Eberhardt, Phys. Rev. B 56, 13147 (1997).

${ }^{5}$ J. A. Carlisle, E. L. Shirley, L. J. Terminello, J. J. Jia, T. A. Callcott, D. L. Ederer, R. C. C. Perera, and F. J. Himpsel, Phys. Rev. B 59, 7433 (1999).

${ }^{6}$ Y. Arakawa, IEEE J. Sel. Top. Quantum Electron. 8, 823 (2002).

${ }^{7}$ S. S. Dhesi, C. B. Stagarescu, K. E. Smith, D. Doppalapudi, R. Singh, and T. D. Moustakas, Phys. Rev. B 56, 10271 (1997).

${ }^{8}$ Y.-C. Chao, C. B. Stagarescu, J. E. Downes, P. Ryan, K. E. Smith, D. Hanser, M. D. Bremser, and R. F. Davis, Phys. Rev. B 59, R15586 (1999).

${ }^{9}$ C. B. Stagarescu, L.-C. Duda, K. E. Smith, J. H. Guo, J. Nordgren, R. Singh, and T. D. Moustakas, Phys. Rev. B 54, R17335 (1996).

${ }^{10}$ K. Lawniczak-Jablonska, T. Suski, I. Gorczyca, N. E. Christensen, K. E. Attenkofer, R. C. C. Perera, E. M. Gullikson, J. H. Underwood, D. L. Ederer, and Z. Liliental Weber, Phys. Rev. B 61, 16623 (2000).

${ }^{11}$ S. Eisebitt, J. Lüning, J.-E. Rubensson, and W. Eberhardt, Phys. Status Solidi B 215, 803 (1999).

${ }^{12}$ V. N. Strocov, T. Schmitt, J.-E. Rubensson, P. Blaha, T. Paskova, and P. O. Nilsson, Phys. Status Solidi B 241, R27 (2004).

${ }^{13} \mathrm{~T}$. Paskova and B. Monemar, in III-Nitride Semiconductors: Growth, edited by O. Manasreh (Taylor \& Francis Group, New York, 2003), pp.175-236.

${ }^{14}$ R. Denecke, P. Väterlein, M. Bässler, N. Wassdahl, S. Butorin, A. Nilsson, J.-E. Rubensson, J. Nordgren, N. Mårtensson, and R. Nyholm, J. Electron Spectrosc. Relat. Phenom. 101-103, 971 (1999).

${ }^{15}$ J. Nordgren, G. Bray, S. Cramm, R. Nyholm, J.-E. Rubensson, and N. Wassdahl, Rev. Sci. Instrum. 60, 1690 (1989).

${ }^{16}$ W. R. L. Lambrecht, S. N. Rashkeev, B. Segall, K. LawniczakJablonska, T. Suski, E. M. Gullikson, J. H. Underwood, R. C. C. Perera, J. C. Rife, I. Grzegory, S. Porowski, and D. K. Wickenden, Phys. Rev. B 55, 2612 (1997).

${ }^{17}$ J. A. Bearden, Rev. Mod. Phys. 39, 78 (1967).

${ }^{18}$ J. P. Perdew, K. Burke, and M. Ernzerhof, Phys. Rev. Lett. 77, 3865 (1996).

${ }^{19}$ E. Sjöstedt, L. Nordström, and D. J. Singh, Solid State Commun. 114, 15 (2000).

${ }^{20}$ G. K. H. Madsen, P. Blaha, K. Schwarz, E. Sjöstedt, and L. Nordström, Phys. Rev. B 64, 195134 (2001).

${ }^{21}$ P. Blaha, K. Schwarz, G. K. H. Madsen, D. Kvasnicka, and J. Luitz, WIEN2k, An Augmented Plane Wave Plus Local Orbitals Program for Calculating Crystal Properties (Vienna University of Technology, Austria, 2001).

${ }^{22}$ J. Luitz, M. Maier, C. Hébert, P. Schattschneider, P. Blaha, K. Schwarz, and B. Jouffrey, Eur. Phys. J. B 21, 363 (2001).

${ }^{23}$ U. von Barth and G. Grossmann, Phys. Rev. B 25, 5150 (1982).

${ }^{24}$ D. A. Muller, D. J. Singh, and J. Silcox, Phys. Rev. B 57, 8181 (1998).

${ }^{25}$ P. Bennich, T. Wiell, O. Karis, M. Weinelt, N. Wassdahl, A. Nilsson, M. Nyberg, L. G. M. Pettersson, J. Stöhr, and M. Samant, 
Phys. Rev. B 57, 9274 (1998).

${ }^{26}$ M. Grioni, J. F. van Acker, M. T. Czyzyk, and J. C. Fuggle, Phys. Rev. B 45, 3309 (1992).

${ }^{27}$ V. N. Strocov, R. Claessen, F. Aryasetiawan, P. Blaha, and P. O. Nilsson, Phys. Rev. B 66, 195104 (2002).

${ }^{28}$ L.-W. Wang, L. Bellaiche, S.-H. Wei, and A. Zunger, Phys. Rev. Lett. 80, 4725 (1998).
${ }^{29}$ E. Calleja, M. A. Sánchez-García, F. J. Sánchez, F. Calle, F. B. Naranjo, E. Muñoz, U. Jahn, and K. Ploog, Phys. Rev. B 62, 16826 (2000); J. Risti, E. Calleja, M. A. Sánchez-García, J. M. Ulloa, J. Sánchez-Páramo, J. M. Calleja, U. Jahn, A. Trampert, and K. H. Ploog, Phys. Rev. B 68, 125305 (2003).

${ }^{30}$ S. M. Lee, Y. H. Lee, Y. G. Hwang, J. Elsner, D. Porezag, and T. Frauenheim, Phys. Rev. B 60, 7788 (1999). 